\title{
Coronavirus Disease (COVID-19) Outbreak and Its Impact on Spinal Daily Practice : Preliminary Report from a Single (Regional) University Hospital in Republic of Korea
}

\author{
Chang Hwa Ham, ${ }^{1}$ Hong Joo Moon, Joo Han Kim, ${ }^{1}$ Youn-Kwan Park, ${ }^{1}$ Tae Hoon Lee, ${ }^{2}$ Woo-Keun Kwon ${ }^{1,3}$ \\ Department of Neurosurgery, Korea University Guro Hospital, Korea University College of Medicine, Seoul, Korea \\ Spine and Joint Center, 29988 Hospital, Seoul, Korea \\ Focused Training Center for Trauma, ${ }^{3}$ Korea University Guro Hospital, Korea University College of Medicine, Seoul, Korea
}

Objective : Since the first discovery of the 2019 novel coronavirus (COVID-19), rapid and wide spread of the disease has been reported and the World Health Organization announced that a 'pandemic' has started. Up to date there is little known regarding the impact of this outbreak on spinal specialists' daily clinical practice. We intended to evaluate how COVID-19 has affected the number of spinal disease patients we meet and operate in daily practice.

Methods : The de-identified data regarding number of patients visiting the spine clinic at a tertiary referral hospital and a secondary level hospital from January, February and March of 2017 to 2020 were retrospectively reviewed. The number of outpatient department (OPD) visits, number of emergency room (ER) visits as well as number of surgeries performed during the reviewed period were collected and analyzed, comparing 2020 to the previous 3 years.

Results : The number of daily OPD visits showed a steady decrease starting from January, and presented a statistically significant decrease by early March 2020, compared to the previous 3 years. During the same period, decrease in number of daily ER visits was statistically significant as well. The number of elective surgeries or number of surgeries for patients admitted via ER during COVID-19 outbreak remained similar to that of 2017-2019 suggesting, despite the decrease of patients visiting the hospital for spinal diseases, those whom required surgery still visited the hospital. The results were consistant among other hospital level.

Conclusion : The outbreak of COVID-19 affected our daily practice as OPD and ER visits reduced but did not affect the number of surgeries. We believe that this report will be informative to spinal specialists worldwide fighting the COVID-19 pandemic.

Key Words : COVID-19 · Coronavirus · Spine · Spinal practice.

\section{INTRODUCTION}

Coronavirus $(\mathrm{CoV})$ is a very common pathogen, which eas- ily affects the human respiratory system. However, this common pathogen has brought several serious outbreaks in the past. In 2002 and 2003, an outbreak of CoV induced respira-

\footnotetext{
- Received : April 14, 2020 •Revised : April 27, 2020 •Accepted : May 4, 2020

- Address for reprints : Woo-Keun Kwon

Department of Neurosurgery, Korea University Guro Hospital, Korea University College of Medicine, 148 Gurodong-ro, Guro-gu, Seoul 08308, Korea Tel : +82-2-2626-3100, Fax : +82-2-863-1684, E-mail : kwontym@gmail.com, ORCID : https://orcid.org/0000-0003-0432-8620
} 
tory disease of zoonotic origin occurred and resulted in a great number of casualties in Asia. This subtype of $\mathrm{CoV}$ infected the host's respiratory system and led to severe acute respiratory syndrome (SARS)-naming the subtype SARS coronavirus (SARS-CoV). In 2012-2013, the appearance of another subtype of $\mathrm{CoV}$ showed global outbreak, which was found to be a new zoonotic origin of $\mathrm{CoV}$ from the Middle-East. This subtype called Middle East respiratory system coronavirus (MERS$\mathrm{CoV}$ ) had great impact on many countries worldwide including the Republic of Korea (R.O.K.), infecting more than 2500 patients.

In December 2019, a number of patients was diagnosed of pneumonia of an unknown etiology in Wuhan, China, and within a short time period, a new $\mathrm{CoV}$ was found to be the etiology of this respiratory viral disease. World health organization (WHO) named this 2019 novel CoV as COVID-19 in February $11 s t, 2020^{16)}$. As early reports predicted its worldwide spread $^{17)}$, it has quickly become a worldwide outbreak.

In R.O.K., the first confirmed case of COVID-19 infection was reported on January 19th of $2020^{8}$. While the first confirmed patient was reported to have arrived from Wuhan, China, the disease started to spread by community transmis$\operatorname{sion}^{10)}$. And within a very short period of time, the number of confirmed patients peaked to 4212 on March 2nd, reported by the Korean Center for Disease control and Prevention $(\mathrm{KCDC})^{11}$. Although the increase of diagnoses has become steady compared to those of early March, the number is still increasing daily and has reached 9661 as of $12 \mathrm{AM}$ on March $31 \mathrm{st}^{11)}$, and 10237 on April $6 \mathrm{th}^{11)}$. While the early cases of COVID-19 were limited to far-east Asia, including China and R.O.K., the recent burst of outbreak is significantly affecting Europe and North America. The diagnosed cases in the U.S. has peaked up to 140904 , and a total of 2405 deaths are reported by March $31 \mathrm{st}^{2}$. This number has increased rapidly and by April 5th, it has reached 304826 cases and 7616 deaths ${ }^{2}$. As we can see in the numbers, confirmed infection cases are growing exponentially every second. Just like many other countries worldwide, the outbreak of COVID-19 has brought great impact to R.O.K., not limited to national health issues but also regarding social, economic and political issues. And by the time of preparing this manuscript, we are still suffering from this situation trying to overcome it.

As spinal specialists, we are often not confronted with patients diagnosed with COVID-19, and as of yet, there is no clear evidence that the COVID-19 has direct impact on any form of spinal disease. However, as the worldwide outbreak of this disease has become a social phenomenon rather than a simple infectious disease, it is true that COVID-19 has already affected or will affect our practice. From a country that has faced this outbreak several weeks earlier than other nations, we have intended to evaluate how this disease has affected the number of spinal disease patients we treat in daily practice and share our results with colleagues worldwide who are likely to face the similar situation in the near future. To our knowledge this is the first report to review the impact of COVID-19 on spinal specialists' practice.

\section{MATERIALS AND METHODS}

\section{Materials}

Waiver of Institutional Review Board (IRB) was approved by the Local Ethical Committee, as the study was designed as a retrospective review of totally de-identified data of patients (Korea University Guro Hospital IRB waiver number : 2020GR0156). The number of patients who visited the spine clinic or the division of spine for spinal disease treatment at a single regional tertiary referral university hospital was reviewed. To reflect the effect of COVID-19 outbreak, the patients who visited the hospital starting from January 1st to March 31st, 2020 were included. The same data starting from January to March of 2017 to 2019 were also collected for comparison with that of 2020. The number of out-patient department (OPD) visits, emergency room (ER) visits was collected as well as the number of surgeries performed (elective or emergency). The classification of diagnoses made according to the international classification of diseases (ICD) codes of each patients was also collected in order to perform subgroup analysis, by dividing each number of visits according to the ICD codes.

The data were also collected from a single local private spine and joint specialized hospital, in order to briefly compare the differences among hospital levels of spinal care. Due to limited resources, the data from the secondary level spine specialized hospital were limited to data from 2019 to 2020.

\section{Statistical analysis}

Data are presented in actual data or as average \pm standard 
deviation. All statistical analyses comparing data from year by year were carried out using the independent student's t-test, Mann-Whitney test or Kruskall-Wallis test as appropriate. SPSS ver. 20.0 (IBM Corporation, Armonk, NY, USA) was used for each analysis and $p$-values less than $0.05(p<0.05)$ were determined to be statistically significant.

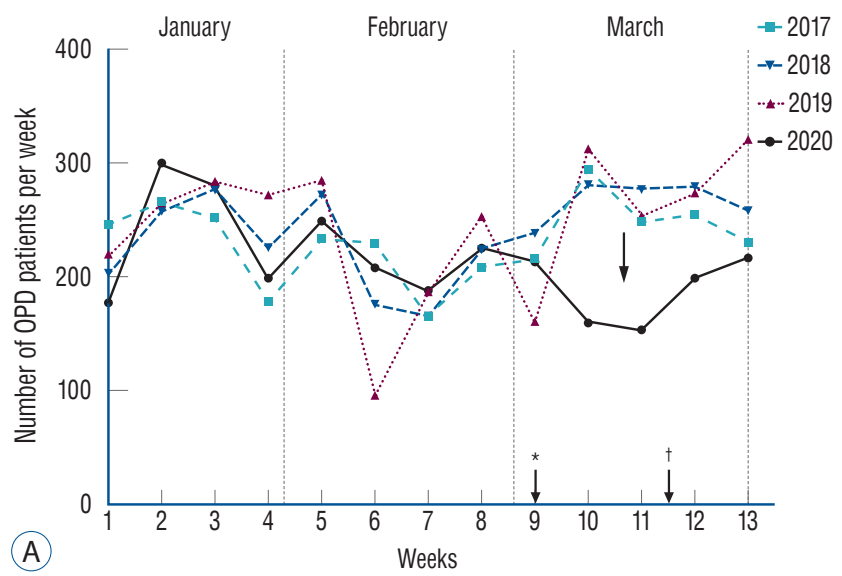

\section{RESULTS}

\section{Number of OPD patients}

The number of daily OPD visits at the tertiary referral hospital spine department on 2017 to 2019 was $52.24 \pm 11.1,54.57 \pm$ 9.14 , and $57.04 \pm 10.03$, respectively; while in 2020 , the number

Fig. 1. Graphs showing the number of OPD visits to the spine center of a tertiary referral center in Republic of Korea during January to March of 20172020. A : Number of weekly OPD patients during the reviewed period. B : Comparison of average OPD visits per day during 2017 to 2020 by month. *Timepoint of COVID-19 alert level raised to highest, 'RED', by the Korean government. 'Timepoint of COVID-19 confirmed as pandemic by WHO. ${ }^{\ddagger} p<0.05$. OPD : out-patient department, COVID-19: 2019 novel coronavirus, WHO : World Health Organization.

Table 1. Number of daily OPD visits and ER visits starting from January 1st to March 31st of 2017-2020

\begin{tabular}{|c|c|c|c|c|c|c|}
\hline & \multicolumn{4}{|c|}{ Tertiary referral hospital } & \multicolumn{2}{|c|}{ Secondary level hospital } \\
\hline & 2017 & 2018 & 2019 & 2020 (COVID-19) & 2019 & 2020 (COVID-19) \\
\hline \multicolumn{7}{|c|}{ Average OPD visits per day } \\
\hline January & $49.05 \pm 9.67$ & $50.38 \pm 13.25$ & $58.19 \pm 8.64$ & $54.04 \pm 8.14$ & & \\
\hline February & $53.67 \pm 10.91$ & $54.50 \pm 9.64$ & $53.00 \pm 7.91$ & $62.25 \pm 6.69$ & & \\
\hline March & $56.75 \pm 11.32$ & $55.95 \pm 13.92$ & $55.29 \pm 16.83$ & $37.68 \pm 15.2^{*}$ & & \\
\hline Total & $52.24 \pm 11.10$ & $54.57 \pm 9.14$ & $57.04 \pm 10.03$ & $47.04 \pm 17.95$ & & \\
\hline \multicolumn{7}{|c|}{ Total OPD visits } \\
\hline January & & & & & 2506 & 2296 \\
\hline February & & & & & 2277 & 1877 \\
\hline March & & & & & 2446 & 1533 \\
\hline Total & & & & & 7229 & 5706 \\
\hline \multicolumn{7}{|c|}{ Average ER visits per day } \\
\hline January & $1.23 \pm 1.06$ & $0.94 \pm 1.26$ & $0.81 \pm 0.87$ & $0.71 \pm 0.97$ & N/A & $N / A$ \\
\hline February & $0.96 \pm 1.11$ & $1.00 \pm 1.02$ & $0.96 \pm 1.23$ & $0.48 \pm 0.74$ & N/A & N/A \\
\hline March & $0.61 \pm 0.71$ & $0.90 \pm 0.83$ & $0.71 \pm 0.97$ & $0.74 \pm 1.00$ & N/A & N/A \\
\hline Total & $0.93 \pm 0.99$ & $0.94 \pm 1.04$ & $0.91 \pm 1.02$ & $0.65 \pm 0.91^{*}$ & $\mathrm{~N} / \mathrm{A}$ & N/A \\
\hline
\end{tabular}

Data from a tertiary referral hospital and from a secondary level spine and joint specialized hospital. Values are presented as mean \pm standard deviation or number (\%). * $p$ value $<0.05$. OPD : out-patient department, ER : emergency room, COVID-19: 2019 novel coronavirus, N/A : not available 

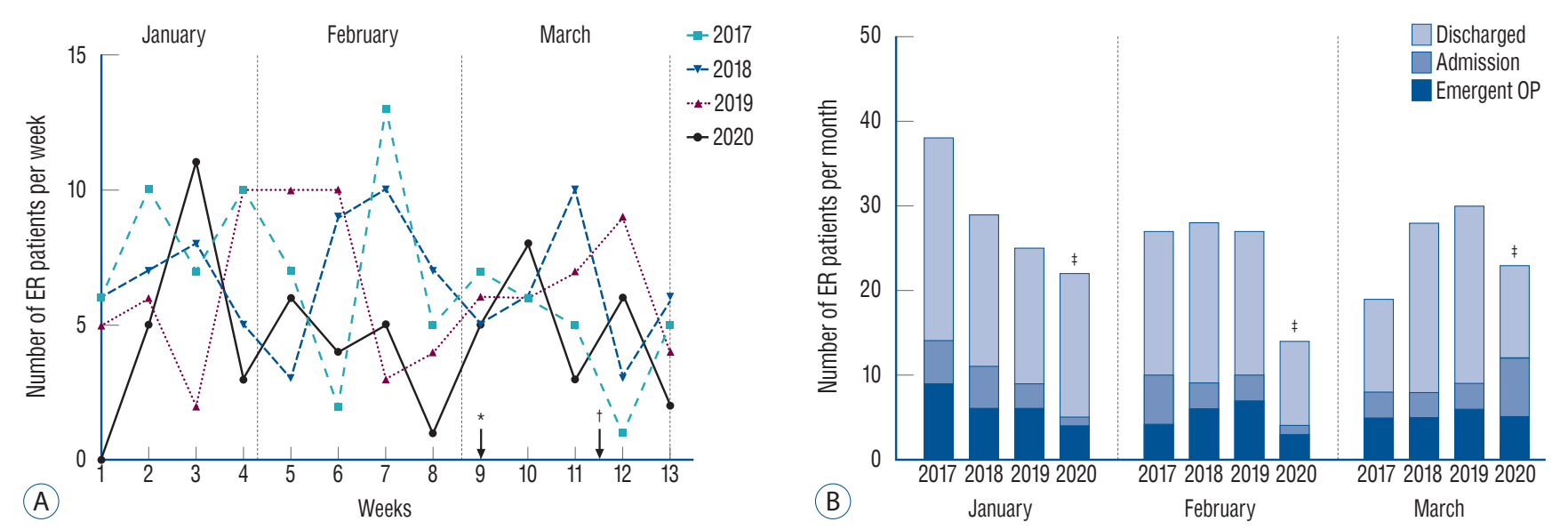

Fig. 2. Graphs showing the number of ER visits to the spine center of a tertiary referral center in Republic of Korea during January to March of 20172020. A : Number of weekly ER patients during the reviewed period. B : Number of monthly ER visits; the number of admissions and emergent surgeries among the ER visits are compared between each year. *Timepoint of COVID-19 alert level raised to highest, 'RED', by the Korean government. ${ }^{\dagger}$ Timepoint of COVID-19 confirmed as pandemic by WHO. ${ }^{~}$ Total number of patients ER patients in 2020 was statistically decreased compared to 2017 and $2018(p<0.05)$, and showed decreased trend compared to 2019 ( $p=0.07)$. ER : emergency, room, OP : operation, COVID-19: 2019 novel coronavirus, WHO : World Health Organization.

of daily visits was $47.04 \pm 17.95$, during the reviewed period. Weekly changes of OPD visits are shown on Fig. 1. While the number of visits of 2020 was steady from week 1 to 8 (January and February), the decrease in number of visits became statistically significant starting from week 9 in early March. Although statistical comparison was impossible due to limited data, the OPD visit numbers showed similar patterns at the secondary level hospital as well. Data are shown on Table 1 and Fig. 1.

\section{Number of ER patients}

The number of daily visits to ER due to spine related degenerative diseases, pains or traumas during 2017 to 2019 was $0.93 \pm$ $0.99,0.94 \pm 1.04$, and $0.91 \pm 1.02$, respectively, whereas it was 0.64 \pm 0.91 in 2020 during the reviewed period. A decrease of daily ER visits was observed during COVID-19 outbreak in 2020. When compared to that of 2019, the number of ER visits showed a decreasing tendency but failed to read statistical significance ( $p=0.07$ ) but the decrease was statistically significant when compared to 2017 or $2018(p<0.05)$. As the secondary hospital we reviewed does not operate an ER, analysis was not available. Data results of ER visits are shown on Table 1 and Fig. 2.

\section{Number of surgeries performed}

The total number of surgeries performed during the re- viewed period was $167,169,147$, and 175 , respectively for 2017 , 2018,2019 , and 2020. The average number of elective surgeries and surgeries for admissions via ER are shown on Table 2 and Fig. 3. The number of total surgeries did not show any statistically significant difference, and the proportion of disease entities were not different as well. The number of surgeries via ER during COVID-19 outbreak showed a decrease on average compared to that of 2017-2019, but was not statistically significant.

\section{DISCUSSION}

As we have shown in the introduction, the spread of COVID-19 has been announced to be a "pandemic" by the $\mathrm{WHO}^{16}$, and it has brought significant changes to every aspect of our daily life.

The symptoms of COVID-19 infections include cough, fever, fatigue, sputum, rhinorrhea, diarrhea or headache etc ${ }^{1,4,13-15}$. Up to date, there is no evidence that this infectious disease has any direct effects on the spine. However, as we have described in the introduction, the outbreak of COVID-19 is rather a social phenomenon than merely a rapidly spreading upper respiratory tract infection. At the early stage COVID-19 outbreak, a spine and joint specialized local private hospital in Seoul, Korea (secondary level hospital) reported a confirmed case of 
Table 2. Total number of surgeries starting from January 1st to March 31st of 2017-2020

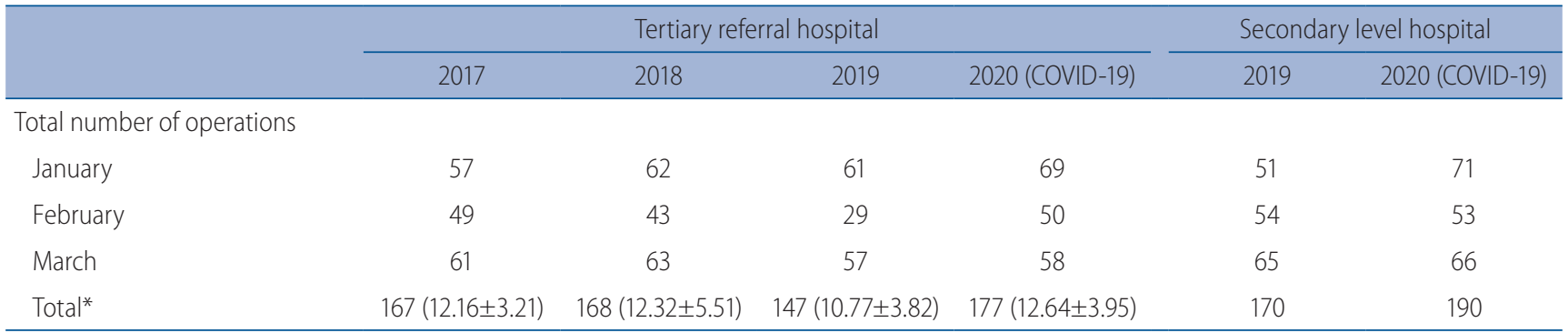

Data from a tertiary referral hospital and a secondary level spine and joint specialized hospital. *Average number of operations per week \pm standard deviation are shown within brackets. COVID-19: 2019 novel coronavirus
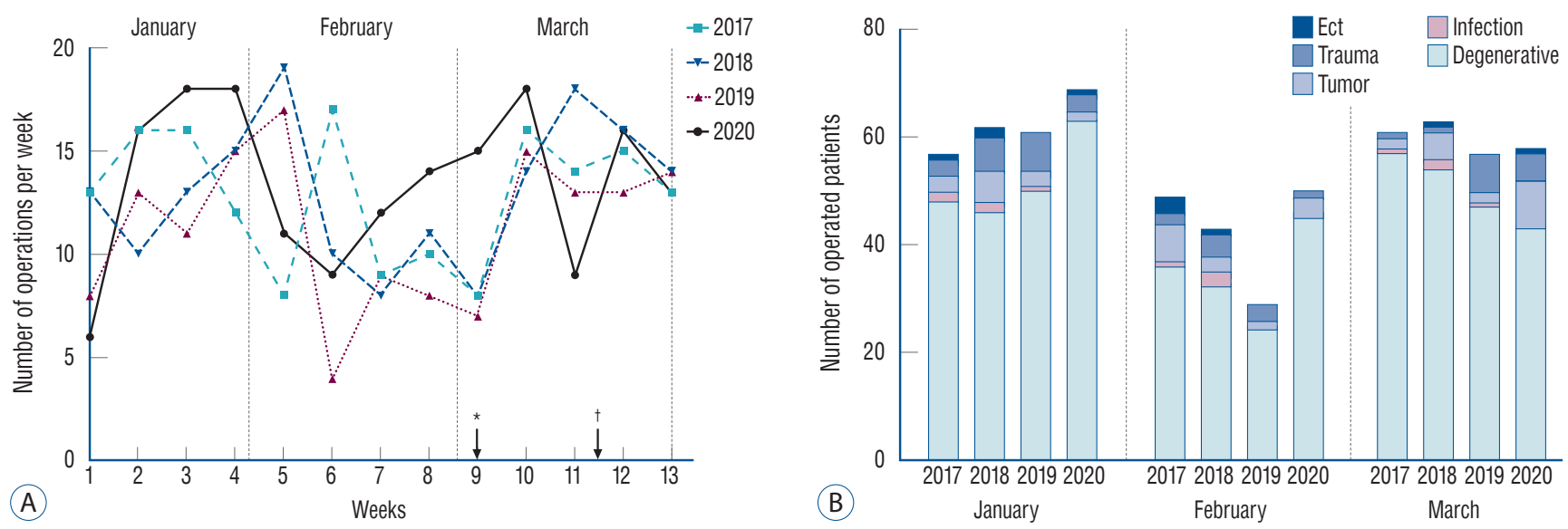

Fig. 3. Graphs showing the number of spine surgeries at a spine center of a tertiary referral center in Republic of Korea during January to March of 2017-2020. A : Number of weekly surgeries by week during the reviewed period. B : Number of monthly surgeries and the proportion of disease entities during the reviewed period. *Timepoint of COVID-19 alert level raised to highest, 'RED', by the Korean government. 'Timepoint of COVID-19 confirmed as pandemic by WHO. COVID-19: 2019 novel coronavirus, WHO : World Health Organization.

COVID-19 infection ${ }^{9}$. A patient who was admitted at this center for a musculoskeletal disease suffered from fever and cough after surgery, and the patient was confirmed to have COVID-19 infection. Immediately after the confirmation of disease, the hospital was cohort isolated, and any further contact was forbidden by the government. The hospital was closed, isolated for 2 weeks and of course clinical practice was stopped during this period unwillingly. As we can see in this case, the infectious organism does not directly affect the spine, but confirmed infection of patients can affect the spinal specialist's practice.

Even in hospitals and spinal clinics with no confirmed cases of COVID-19, we noticed a significant decrease of spinal patients visiting us for treatment. As we have shown in the results, the number of patients visiting the OPD of spinal clinics has significantly decreased both at a tertiary medical center and at a private spine/joint specialized hospital. The number of OPD visits dropped significantly in March, when compared with the average number of OPD visits during the same period of previous 3 years. In late February, as the Korean Government raised the national COVID-19 threat alert level to highest, 'RED'3), the decrease became statistically significant. This result suggests that the outbreak of COVID-19 has significantly affected the patient's decision to visit hospitals via OPD. Although we do not have any survey results or any other evidence on which factors have led us to this result so far, we suppose that the fear of getting exposed to COVID-19 infection by any chance has kept the patients home rather than visiting a hospital. It has been reported that the fear against a certain phenomenon can act as a factor discouraging the patients to visit hospitals ${ }^{6}$. This pattern of decrease in OPD patients was similar among different hospital levels.

Interestingly, like the change of OPD visit numbers, the number of patients visiting the ER due to spinal diseases also 
presented a significant decrease as shown in results. When preparing this manuscript, we hypothesized the number of OPD patients with spine related diseases could decrease due to the outbreak of COVID-19 and thought that the number of ER visits would remain the same. However, the results were not as we expected, and the number of ER visits also decreased. The general population may have advocated the government's recommendations of social distancing, avoiding hospital visits unless inevitable and resulting in a decreased OPD and ER visits.

Despite the significant decrease in OPD and ER visits, the average number of both elective and emergency surgeries during COVID-19 outbreak was maintained compared to that of the same period in 2017-2019. This is an important point of our results that the number of elective and emergency operations during COVID-19 outbreak was not lower compared to the previous 3 consecutive years, which suggests that patients with severe pain, neurologic deficits or severe trauma requiring surgical intervention still visited the hospital regardless of the COVID-19 outbreak, and underwent surgery. We assume that this could be a possible reason that has led to the maintained number of elective and emergency operations while hospital visits via OPD and ER both decreased. By scrutinizing our results, we noticed that the COVID-19 outbreak has brought significant changes into our clinical practice. It led to a significant decrease in number of patients we meet both at OPD and ER, however did not affect the number of surgeries we perform.

Three months have passed since the outbreak of COVID-19 and we still do not have an evidence based, scientifically confirmed protocol for COVID-19 prevention at a health care providing center level. However, codes of conducts from the government and other scientific committees are being introduced. The KCDC recommends three instructions for health care providers, and six behavioral recommendations for the general population ${ }^{5,12)}$. The three instructions that health care providers regardless of their medical specialty should follow are presented on Table 3. These instructions are, of course, not only for those dealing with infectious disease, but also the same for general physicians, including spinal specialists. And all spine specialists in our center are also following them. The six behavioral recommendations for general population are also shown on Table 3.

During the investigation period, we had a single case of suspected COVID-19 patient presenting with fever, who was fortunately confirmed to be negative by the diagnostic test. The patient was admitted for an elective lumbar spinal fusion surgery and presented with fever of unknown origin on the operation day, after arriving at the operating theater. The patient did not have any confirmed evidence of infection nor any traveling history to dangerous regions. Nevertheless, following strict regulations of the hospital regarding how to deal with fever patients under the recent circumstances, the elective surgery was immediately cancelled and the patient was referred

Table 3. Recommendations for health care providers and general population under COVID-19 outbreak, by the Korean Center for Disease control and Prevention

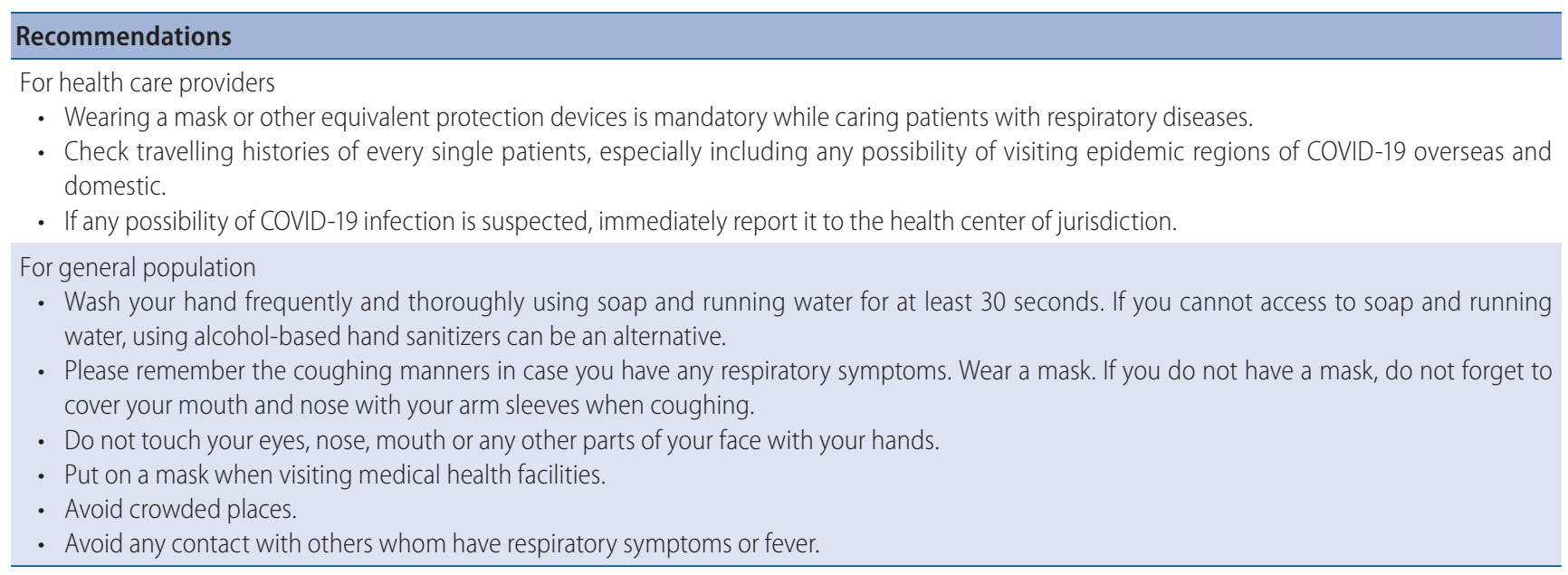

COVID-19: 2019 novel coronavirus 
for COVID-19 screening tests. Only after final confirmation of negative results, the patient was allowed to undergo the surgery which was 2 days after the initially scheduled date. The Korean association of anesthesiologists has announced a recommendation for institutions or hospitals regarding peri-operative patients suspected of COVID-19 $9^{7}$, and this guideline helped us to promptly respond to this unexpected event. This guideline has detailed instructions for health care providers dealing with peri-operative patients, starting from transport of patients, managements of the operating theater to the anesthesia process ${ }^{7}$.

Our report has certain limitations. As this is an early preliminary report from a single regional university hospital center, the major weakness of this study is the small number of cohort and it is hard to say that our data represents the national data. As we are still encountering the outbreak situation, conducting a larger study involving multiple centers was challenging and the data is collected from a single institution of each level of hospitals. To minimize this weakpoint, we reviewed the data from two different level hospitals. Nevertheless, we believe that our data provides us with a clue on what happens under infectious disease outbreaks. After the outbreak subsides and the disease is under control, further larger studies are guaranteed, and additional data from the public national health services may add strength to such studies.

Another limitation is the lack of evidence based information regarding the significant reasons as to why patients avoided visits to the OPD spinal clinic, and it is a conjecture in this report that the fear for COVID-19 may have been the cause. Under current circumstances, we were unable to get surveys from patients or clinical health care providers. This as well can be of object for future studies. Furthermore, our study fails to reflect the complex epidemiologic factors which may affect the number of OPD or ER visits under these situations. For example, regional factors, hospital factors, physician factors, patient factors (pain scores, neurologic status or detailed disease severity), socioeconomic factors, etc. should be considered in further studies. Under these limited circumstances, we believe that it is reasonable to assume that the significant change in patient visits is strongly related to the fear of COVID-19 outbreak and the government's recommendations to refrain from outside activities. Despite these limitations, as a first narrative report on this issue, this article will help us understand what happens to our practice and be pre- pared for the change.

To our knowledge this is the very first clinical article to report the impact of COVID-19 outbreak on change of spinal specialists' practice, and we have confidence that this report will be informative to spinal specialists, pain physicians and clinical neurology/neurosurgery practitioners. As the R.O.K. has met this outbreak several weeks earlier than other nations, we hope this information could help our colleague spinal specialists worldwide and give them a chance to get prepared to the possible impact on their practice, which will come in the near future. We also provided the recommended instructions for both health care providers and general population we are following in R.O.K., and hope this information helps readers.

\section{CONCLUSION}

By retrospectively reviewing de-identified data of spinal clinic patients at a regional university hospital, we discovered that the outbreak of COVID-19 affected our daily practice. The OPD and ER visits during the reveiwed period reduced but number of surgeries were not affected. We hope that this report will be informative to spinal specialists worldwide fighting the COVID-19 pandemic.

\section{CONFLICTS OF INTEREST}

No potential conflict of interest relevant to this article was reported.

\section{INFORMED CONSENT}

This type of study does not require informed consent.

\section{AUTHOR CONTRIBUTIONS}

\author{
Conceptualization : $\mathrm{CHH}$, WKK \\ Data curation : CHH, HJM, JHK, YKP, THL, WKK \\ Formal analysis : $\mathrm{CHH}, \mathrm{WKK}$ \\ Methodology : CHH, HJM, JHK, YKP, THL, WKK \\ Project administration : $\mathrm{CHH}$, WKK
}




\author{
Visualization : CHH, HJM, JHK, YKP, THL, WKK \\ Writing - original draft : $\mathrm{CHH}, \mathrm{WKK}$ \\ Writing - review \& editing: CHH, HJM, JHK, YKP, THL, \\ WKK
}

\section{ORCID}

$\begin{array}{ll}\text { Chang Hwa Ham } & \text { https://orcid.org/0000-0003-1020-4210 } \\ \text { Hong Joo Moon } & \text { https://orcid.org/0000-0002-3841-9080 } \\ \text { Joo Han Kim } & \text { https://orcid.org/0000-0002-4747-9763 } \\ \text { Youn-Kwan Park } & \text { https://orcid.org/0000-0003-0501-3540 } \\ \text { Tae Hoon Lee } & \text { https://orcid.org/0000-0002-4673-4689 } \\ \text { Woo-Keun Kwon } & \text { https://orcid.org/0000-0003-0432-8620 }\end{array}$

\section{References}

1. Carlos WG, Dela Cruz CS, Cao B, Pasnick S, Jamil S : Novel Wuhan (2019nCoV) coronavirus. Am J Respir Crit Care Med 201 : P7-P8, 2020

2. Centers for Disease Control and Prevention : Disease 2019 (COVID-19): Cases in U.S.. Available at : https://www.cdc.gov/coronavirus/2019-ncov/ cases-updates/cases-in-us.html

3. Chu $M$, Jung $Y S$ : Coronavirus spread in South Korea surges to 763 cases and 7 deaths. Avaliable at : http://www.monews.co.kr/ news/articleView.html?idxno $=208524$

4. Huang $C$, Wang $Y$, Li $X$, Ren L, Zhao J, Hu Y, et al. : Clinical features of patients infected with 2019 novel coronavirus in Wuhan, China. Lancet 395 : 497-506, 2020

5. Huh $\mathrm{S}$ : How to train the health personnel for protecting themselves from novel coronavirus (COVID-19) infection during their patient or suspected case care. J Educ Eval Health Prof 17 : 10, 2020

6. Hursitoglu M, Erdal GS, Akdeniz E, Kocoglu H, Kocamaz N, Tevetoglu IO, et al. : Early effect of the fear of terrorism on a hospital's emergency department use and on the incidence of cardiovascular events: a Turkish multicenter study. Int J Health Plann Manage 34 : 872-879, 2019
7. Kim HJ, Ko JS, Kim TY; Scientific Committee of the Korean Society of Anesthesiologists : Recommendations for anesthesia in patients suspected of COVID-19 coronavirus infection. Korean J Anesthesiol 73 : 89-91, 2020

8. Kim JY, Choe PG, Oh Y, Oh KJ, Kim J, Park SJ, et al. : The first case of 2019 novel coronavirus pneumonia imported into Korea from Wuhan, China: implication for infection prevention and control measures. J Korean Med Sci 35 : e61, 2020

9. Ko SJ : Corona Virus at a spine and joint specialized hospital. Lessons from the case. Avaliable at : https://www.doctorsnews. co.kr/news/articleView.html?idxno=133217

10 Korean Society of Infectious Diseases; Korean Society of Pediatric Infectious Diseases; Korean Society of Epidemiology; Korean Society for Antimicrobial Therapy; Korean Society for Healthcare-associated Infection Control and Prevention; Korea Centers for Disease Control and Prevention : Report on the epidemiological features of Coronavirus disease 2019 (COVID-19) outbreak in the Republic of Korea from January 19 to March 2, 2020. J Korean Med Sci 35 : e112, 2020

11. Ministry of Health and Welfare : Daily update of coronavirus-19 cases in Korea. Avaliable at : http://ncov.mohw.go.kr/en

12. Ministry of Health and Welfare: Recommendations from KCDC. Avaliable at : http://ncov.mohw.go.kr/infoBoardList.do

13. Ren LL, Wang YM, Wu ZQ, Xiang ZC, Guo L, Xu T, et al. : Identification of a novel coronavirus causing severe pneumonia in human: a descriptive study. Chin Med J (Engl) 133 : 1015-1024, 2020

14. Rothan HA, Byrareddy SN : The epidemiology and pathogenesis of coronavirus disease (COVID-19) outbreak. J Autoimmun 109 : 102433, 2020

15. Wang W, Tang J, Wei F : Updated understanding of the outbreak of 2019 novel coronavirus (2019-nCoV) in Wuhan, China. J Med Virol 92 : 441-447, 2020

16. World Health Organization : Coronavirus disease (COVID-19) Pandemic. Available at : https://www.who.int/emergencies/diseases/novelcoronavirus-2019

17. Zhao S, Lin Q, Ran J, Musa SS, Yang G, Wang W, et al. : Preliminary estimation of the basic reproduction number of novel coronavirus (2019$\mathrm{nCoV}$ ) in China, from 2019 to 2020: a data-driven analysis in the early phase of the outbreak. Int J Infect Dis 92 : 214-217, 2020 